\title{
Massive MIMO Systems for 5G
}

\author{
Created by: Robin $^{\text {Chataut }}{ }^{1}$, Robert Akl ${ }^{2}$ \\ 1, Department of Computer Science and Engineering University of North Texas \\ Denton, Texas, USA; robin.chataut@unt.edu \\ 2, Department of Computer Science and Engineering University of North Texas \\ Denton, Texas, USA; Robert.AkI@unt.edu
}

Version received: 23 June 2020

check for updates

The global bandwidth shortage in the wireless communication sector has motivated the study and exploration of wireless access technology known as massive Multiple-Input Multiple-Output (MIMO). Massive MIMO is one of the key enabling technology for next-generation networks, which groups together antennas at both transmitter and the receiver to provide high spectral and energy efficiency using relatively simple processing. Obtaining a better understating of the massive MIMO system to overcome the fundamental issues such as pilot contamination, channel estimation, precoding, user scheduling, energy efficiency, and signal detection is vital for the successful deployment of $5 \mathrm{G}$ and beyond networks. Some of the recent trends in massive MIMO are terahertz communication, ultra massive MIMO (UM-MIMO), visible light communication (VLC), machine learning, and deep learning.

With globalization, present-day networks are facing high traffic demands, and to fulfill these needs, cellular systems are deployed within a few hundred-meter distances, and wireless Local Area Networks (LAN) are placed almost everywhere. Along with increased mobile broadband service, the introduction of new concepts like the Internet of Things (IOT) and Machine-to-Machine Communication (M2M) are also contributing to the increased wireless traffic. The global deployment of cellular service cultivates the cell phone users to be used to the mobile data in their day to day life tremendously. The services like video calling, online gaming, social media applications like Facebook, Twitter, WhatsApp, have changed our life drastically with the capabilities of the third-generation (3G), fourth-generation (4G), and fifth-generation (5G) networks, like lower latency and high data rate ${ }^{[1]}$. A full cell phone connected world is expected in the next few years, which will be mainly characterized by growth in users, connectivity, data traffic volume, and a wide range of applications. In the next few years, technology like augmented reality, virtual reality, ultra high definition video, 3D video, and features like a mobile cloud will become popular to enrich the ultimate user experience. From 2017-2022, smartphone traffic is expected to increase by ten times, and overall, mobile traffic will be increased by eight times ${ }^{[2]}$. Figure 1 shows the growth in mobile data traffic and the number of connected devices from 2017-2022 [3] . By the end of 2022, more than 90 percent of the traffic will come from cell phones. This colossal amount of mobile data traffic is challenging to manage with the capabilities of previous wireless generation systems.

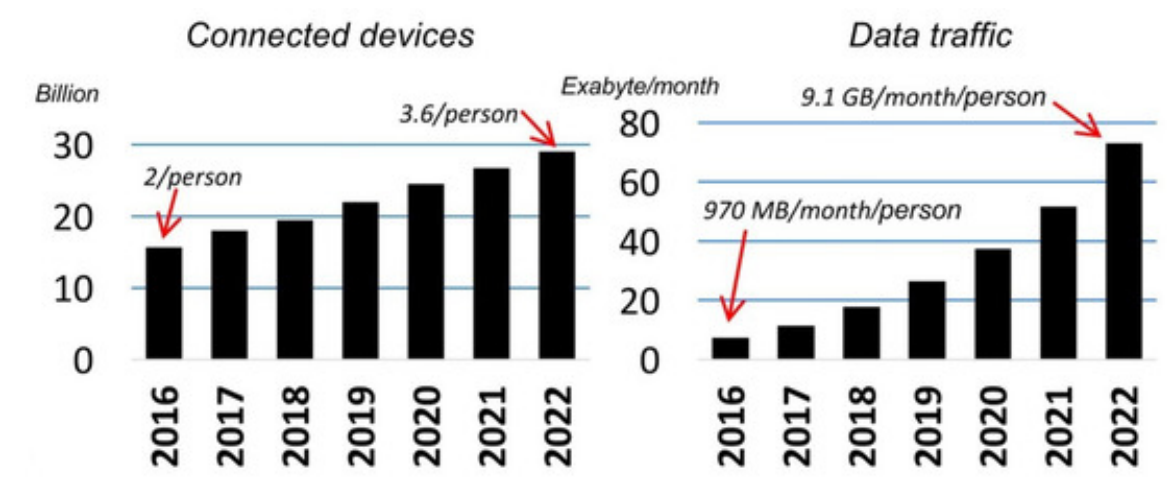

Figure 1. Global mobile data traffic and growth in connected devices from 2017 to 2022. 
The primary issue with the ongoing development of the wireless network is that it is dependent upon either increasing bandwidth (spectrum) or densifying the cells to achieve the required area throughput. These resources are rare and are reaching their saturation point within a few years. Also, increasing bandwidth or densifying the cells increases the cost of the hardware and increases latency. The third factor, which can improve area throughput, that is, spectral efficiency, has remained mostly untouched and unchanged during this rapid development and growth of the wireless network. An efficient wireless access technology that can increase the wireless area throughput without increasing the bandwidth or densifying the cell is essential to achieve the ongoing demands faced by the wireless carriers.

Massive Multiple-Input Multiple-Output (MIMO) is the most enthralling wireless access technology to deliver the needs of $5 \mathrm{G}$ and beyond networks. Massive MIMO is an extension of MIMO technology, which involves using hundreds and even thousands of antennas attached to a base station to improve spectral efficiency and throughput. This technology is about bringing together antennas, radios, and spectrum together to enable higher capacity and speed for the incoming 5G [4]] $\underline{5}]$. The capacity of massive MIMO to increase throughput and spectral efficiency has made it a crucial technology for emerging wireless standards []ㅡㅁ]. The key here is the considerable array gain that massive MIMO achieves with a large number of antennas []. Massive MIMO is a key enabling technology for $5 \mathrm{G}$ and beyond networks, and as intelligent sensing system primarily rely on $5 \mathrm{G}$ and beyond networks to function, massive MIMO and intelligent sensing system are inextricably linked. The data collection from the large number of smart sensors using traditional multi-access schemes is very impractical as it leads to excessive latency, low data rate, and reduced reliability. Massive MIMO with huge multiplexing gain and beamforming capabilities can sense data from concurrent sensor transmission with much lower latency and provide sensors with higher data rates and reliable connectivity. Massive MIMO systems will perform a crucial role to allow information gathered through smart sensors to be transmitted in real-time to central monitoring locations for smart sensor applications such as an autonomous vehicle, remote healthcare, smart grids, smart antennas, smart highways, smart building, and smart environmental monitoring.

\section{References}

1. Young, G.O. Synthetic structure of industrial plastics. In Plastics, 2nd ed.; Peters, J., Ed.; McGraw-Hill: New York, NY, USA, 1964; Volume 3, pp. 15-64. [Google Scholar]

2. Cisco Visual Networking Index: Forecast and Trends, 2017-2022, White Paper c11-741490-00. Available online: https://www.cisco.com/c/en/us/solutions/collateral/service-provider/visual-networking-index-vni/white-paper-c11741490.pdf (accessed on 20 January 2020).

3. Cisco Visual Networking Index: Global Mobile Data Traffic Forecast Update, 2017-2022, White Paper C11-738429-01. Available online: https://www.cisco.com/c/en/us/solutions/collateral/service-provider/visual-networking-indexvni/white-paper-c11-738429.pdf (accessed on 20 January 2020).

4. A Closer Look at Massive MIMO. Available online: https://business.sprint.com/blog/massive-mimo (accessed on 20 January 2020).

5. Rusek, F.; Persson, D.; Lau, B.K.; Larsson, E.G.; Marzetta, T.L.; Edfors, O.; Tufvesson, F. Scaling up MIMO: Opportunities and Challenges With Very Large Arrays. IEEE Signal Process. Mag. 2013, 30, 40-60. [Google Scholar] [CrossRef]

6. Larsson, E.G.; Tufvesson, F.; Edfors, O.; Marzetta, T.L. Massive MIMO for Next Generation Wireless Systems. IEEE Commun. Mag. 2014, 52, 186-195. [Google Scholar] [CrossRef]

7. Marzetta, T.L. Massive MIMO: An Introduction. Bell Labs Tech. J. 2015, 20, 11-22. [Google Scholar] [CrossRef]

8. Wu, X.; Beaulieu, N.C.; Liu, D. On Favorable Propagation in Massive MIMO Systems and Different Antenna Configurations. IEEE Access 2017, 5, 5578-5593.

\section{Keywords}

5G; 6G; beamforming; channel estimation; massive MIMO; millimeter waves; pilot contamination; signal detection; spectral efficiency; terahertz spectrum

(c) 2020 by the author(s). Distribute under a Creative Commans CC BY license 